\title{
A case study on occupational back-support exoskeletons versatility in lifting and carrying
}

\author{
Tommaso Poliero* \\ tommaso.poliero@iit.it \\ Department of Advanced Robotics, \\ Istituto Italiano di Tecnologia \\ Genoa, Italy \\ Department of Informatics \\ Bioengineering Robotics and Systems \\ Engineering, University of Genoa \\ Genoa, Italy \\ Christian Di Natali \\ Department of Advanced Robotics, \\ Istituto Italiano di Tecnologia \\ Genoa, Italy \\ christian.dinatali@iit.it \\ Francesco Draicchio \\ Department of Occupational and \\ Environmental Medicine, \\ Epidemiology and Hygiene, INAIL \\ Monte Porzio Catone, Rome, Italy \\ f.draicchio@inail.it
}

\author{
Matteo Iurato* \\ matteo.iurato@iit.it
}

Department of Informatics

Bioengineering Robotics and Systems

Engineering, University of Genoa

Genoa, Italy

Department of Advanced Robotics, Istituto Italiano di Tecnologia Genoa, Italy

Stefano Toxiri

Department of Advanced Robotics, Istituto Italiano di Tecnologia

Genoa, Italy

stefano.toxiri@iit.it

\author{
Luigi Monica \\ Department of Technological \\ Innovation and Safety Equipment, \\ INAIL \\ Rome, Italy \\ l.monica@inail.it
}

\author{
Matteo Sposito \\ Department of Advanced Robotics, \\ Istituto Italiano di Tecnologia \\ Genoa, Italy \\ matteo.sposito@iit.it \\ Department of Electronics, \\ Information and Bioengineering, \\ Politecnico di Milano \\ Milan, Italy \\ Sara Anastasi \\ Department of Technological \\ Innovation and Safety Equipment, \\ INAIL \\ Rome, Italy \\ s.anastasii@inail.it \\ Darwin G. Caldwell \\ Department of Advanced Robotics, \\ Istituto Italiano di Tecnologia \\ Genoa, Italy \\ darwin.caldwell@iit.it
}

\author{
Vittorio Sanguineti \\ Department of Informatics \\ Bioengineering Robotics and Systems \\ Engineering, University of Genoa \\ Genoa, Italy \\ vittorio.sanguineti@unige.it
}

\author{
Jesús Ortiz \\ Department of Advanced Robotics, \\ Istituto Italiano di Tecnologia \\ Genoa, Italy \\ jesus.ortiz@iit.it
}

\begin{abstract}
To prevent workers performing manual material handling from developing musculoskeletal disorders, occupational back-support exoskeletons are becoming more and more common in industrial scenarios. However, while their beneficial effects on weight lifting tasks are known, additional work is still needed to make them effective in assisting - or at least not hindering - other types of tasks, such as carrying. Therefore, the work presented here studies the effects that varying the assistive strategy according to the performed task, namely exoskeleton versatility, has on the efficacy and

${ }^{*}$ Both authors equally contributed to this research.

Permission to make digital or hard copies of part or all of this work for personal or classroom use is granted without fee provided that copies are not made or distributed for profit or commercial advantage and that copies bear this notice and the full citation on the first page. Copyrights for third-party components of this work must be honored.

For all other uses, contact the owner/author(s).

PETRA 2021, June 29-fuly 2, 2021, Corfu, Greece

(C) 2021 Copyright held by the owner/author(s)

ACM ISBN 978-1-4503-8792-7/21/06.

https://doi.org/10.1145/3453892.3453901
\end{abstract}

the dynamic fit of these devices. After presenting relevant metrics for this analysis, an exemplification of a single-subject case study is detailed. In this study XoTrunk, a back-support exoskeleton, is used to provide assistance during the execution of a complex task involving both lifting and carrying. Results show that exploiting versatility, the control strategy appears to be more natural and respectful of the subject's movements.

\section{CCS CONCEPTS}

- Hardware $\rightarrow$ Hardware validation; $\bullet$ Human-centered computing $\rightarrow$ Human computer interaction (HCI); Interaction design; • Computing methodologies $\rightarrow$ Artificial intelligence.

\section{KEYWORDS}

back-support exoskeletons, occupational exoskeletons; human activity recognition, exoskeleton versatility, lifting, carrying

ACM Reference Format:

Tommaso Poliero, Matteo Iurato, Matteo Sposito, Christian Di Natali, Stefano Toxiri, Sara Anastasi, Francesco Draicchio, Luigi Monica, Darwin G. 
Caldwell, Vittorio Sanguineti, and Jesús Ortiz. 2021. A case study on occupational back-support exoskeletons versatility in lifting and carrying. In The 14th PErvasive Technologies Related to Assistive Environments Conference (PETRA 2021), fune 29-fuly 2, 2021, Corfu, Greece. ACM, New York, NY, USA, 8 pages. https://doi.org/10.1145/3453892.3453901

\section{INTRODUCTION}

Musculoskeletal disorders (MSDs) are a very frequent work-related disease. Despite the introduction in workplaces of various solutions aimed at mitigating the exposure to risk factors (e.g., lift-assist or robotic manipulators) [23], the prevalence of MSDs has not decreased correspondingly [4]. This might be linked with difficulties in new technologies adoption and acceptance. One valid solution to this problem is represented by occupational back-support exoskeletons, a wearable technology capable of reducing physical loading on the lumbar spine when performing lifting tasks, [21]. Indeed, in [5], it has been shown that back-support exoskeletons are able to reduce back-muscle activity by up to $40 \%$ during lifting. This reduction implies a decompression of the lumbar spine and also being lifting frequently executed by workers performing manual material handling $(\mathrm{MMH})$ activities - a mitigation of the potential MSD onset.

Exoskeletons can be passive, quasi-passive or active, depending on the actuation principle. Passive exoskeletons rely, as suggested by the name, on energetically passive components (such as coil or gas springs, elastic bands or flexible beams) in order to generate assistive forces or torques. On the contrary, active exoskeletons use an external source of energy (typically batteries) which powers the actuator(s) (e.g. electrical motor or pneumatic actuators) used to generate the desired forces or torques. Quasi-passive devices, instead, represent a hybrid solution, taking advantage of active elements such as electro-magnetic clutches to decide when to engage the passive elements. A similar description has been presented in [21]. Because of actuation choices, passive exoskeletons share a strong connection with the movement that the wearer is performing. Given the prevalence of lifting activities in $\mathrm{MMH}$ tasks and the high risk of injuries associated with this activity [4], passive exoskeletons are designed and optimized specifically for this lifting movements. Additionally, even though the usage of active actuators introduces less bonds with the movement performed by the wearer, the design of active exoskeletons is still optimized specifically for lifting. However, lifting is not the only task performed during $\mathrm{MMH}$. As an example, after lifting a load, a worker may need to carry the load to another place. Therefore, in order to be successfully adopted by the final users, exoskeletons should be able not only to assist lifting, but also other MMH tasks like carrying, walking, standing, pushing or pulling. If it is not possible to assist these additional tasks, the exoskeleton designers should take care that the device is not hindering when performing non lifting tasks. This is not the case of passive exoskeletons, as reported in [1], where the device assists with lifting activities but hinders walking. On the other hand, active and quasi-passive exoskeletons have the potential to deliver different assistive profiles according to the task being performed, but some sort of activity recognition is required. This latter can rely on user input [21] or be automatic, exploiting human activity recognition (HAR) algorithms, [3], [13], [14], [8].

\subsection{Motivation}

Our previous study [12] on the active exoskeleton XoTrunk showed that the effects of assisting with carrying activities - exploiting the same control strategy employed for lifting - is not ideal, as it restricts the natural gait. It follows that, when broadening the number of tasks for which an exoskeleton is evaluated, the choice of the most informative metrics might be non trivial. For such reason, this work proposes an approach for the evaluation of exoskeleton versatility when performing a complex task. Hereafter, the authors refer to exoskeleton versatility as the ability to recognize which activity the user is performing and provide assistance accordingly. Generally, in the evaluation of back-support exoskeletons it is quite common to focus the analysis predominantly on the efficacy of these devices, [7]. Efficacy is usually proved by showing the ability of the exoskeletons to reduce the activation of the muscles involved in the task. However, it is also important to understand how the exoskeleton and its wearer move and interact with each other. This type of study is defined also dynamic fit, [18]. The objective of this work is to provide a methodology for assessing how exoskeleton versatility might influence not only efficacy but also dynamic fit. In particular, Section 2.1 presents relevant metrics that can be used for this purpose. Then, the performance obtained in an experimental case study involving an active exoskeleton are presented, as an example, in Section 2.2. Specifically, Section 2.2.1 describes the task chosen for the experiment, Section 2.2.2 and Section 2.2.3 introduce the user to data acquisition and processing and, eventually, Section 2.2.4 presents XoTrunk, the exoskeleton used in this assessment. Results and Discussions are presented in Section 3 and Section 4 focusing firstly on efficacy and, then, on dynamic fit.

\section{MATERIALS AND METHODS}

Generally, to prove the efficacy of back-support exoskeletons, the emphasis is on their ability to reduce muscle activation, as reported in [5] and [19]. In this work, to focus on the effects of versatility, it is proposed that not only the exoskeleton efficacy should be evaluated, but also its dynamic fit. Accordingly, the analysis is divided into two parts. Furthermore, it is also shown how this analysis can be adopted not only for lifting tasks, generally addressed in most of the studies [7], but also for different ones. As an example, in Section 2.2, a case study dealing with lifting and carrying tasks is presented.

\subsection{Assessment metrics}

2.1.1 Efficacy. Back-support exoskeletons have their main effect on the muscles responsible for the back extension, namely the Erector Spinae (ES) muscles. Therefore, to evaluate the efficacy of these devices the standard approach is, firstly, to replicate the working scenario in the lab and, then, measure to what extent the exoskeleton usage reduces the activation of these muscles. Common metrics are:

- $90^{\text {th }}$ percentile of EMG signal distribution: in the literature [9], this metric is indicated as being more informative than maximum muscle activation and is traditionally associated with traumatic damages in the inter-vertebral discs. In fact, muscle activity is linked with lumbar compression forces [2], that are responsible of such damages when they exceed a certain threshold [11]. 
- $50^{\text {th }}$ percentile of EMG signal distribution: in the literature [9], this metric is often used to describe how the muscle has been working during the whole recording period and, thus, can be associated with a risk related to repetitive or cumulative fatigue.

Even though back-support exoskeletons have their main effect on the ES, it might be interesting to analyze how they affect other muscles (like hip flexor/extensors) or core stability (abdominal muscles), as done in [1] and [12]. Indeed, this additional investigation can help understanding whether the exoskeleton is generating side effects, such as an increase of the muscle activity in other body regions. So far, many studies [5], [7] have assessed the ability of back-support exoskeletons to reduce ES activation during lifting. However, because of their versatility, defined as the ability to recognize which activity the user is performing and provide assistance accordingly, back-support exoskeletons are expected to have effects also on other relevant $\mathrm{MMH}$ activities.

2.1.2 Dynamic fit. As introduced in [18], dynamic fit is about how the exoskeleton and its wearer move and interact with each other. There are several factors that can be taken into account. Here, the spotlights are on the final user's capability to continue working in a natural way and receive appropriate assistance, according to the task. Indeed, up to date, studies have mainly focused on lifting, but if current exoskeleton control strategies are employed also in other tasks, the assistance effects might be sub-optimal or undesired, as discussed in [12]. Therefore in this work, to show how exoskeleton versatility can improve dynamic fit, the following metrics are proposed:

- Kinematics: to assess whether workers are experiencing constraints in the movements, useful metrics are (i) Time required to Complete the Task (TCT) and (ii) Range of Motion (RoM) of joints like trunk, hip and knee. Moreover, it is worth pointing out that RoMs might be influenced by trajectory changes and, so, also (iii) joint angles average profiles should be considered.

- Intuitiveness and ease of use: to evaluate these aspects, it should be checked if the exploitation of exoskeleton versatile capabilities is intuitive and easy to use. As an example, requiring the user to manually switch between different control strategies might not be the best solution when performing $\mathrm{MMH}$ activities and, thus, automatic switching could be preferred. On the contrary, automatic could be worst than manual switching if it is not working properly. Those as pects can be assessed through questionnaires - relying on well established tests like SUS or Nasa TLX, or on custom defined ones [15], [16].

- Activity recognition performance: the first check should be on the device or the user's capability to recognize the activities being performed. Secondly, the assessment should consider how long it takes for the device, or the user, to recognize that a given activity is being performed. Relevant metrics to answer these questions are represented by confusion matrices $(\mathrm{CM})$ that identify precision, recall and accuracy, and by the estimation-time delay.
Versatile exoskeletons are expected to deliver different assistive profiles according to the performed task and, thus, the above mentioned metrics should be evaluated for each of the user activities. Therefore, the design of an experimental set-up for assessing exoskeletons performance in complex tasks (like those involving both lifting and carrying), should segment the acquired signals according to the performed activity.

\subsection{Case study}

2.2.1 Task description. An experiment, approved by the Ethics Committee of Liguria (protocol reference number: CER Liguria $001 / 2019$ ), was conducted on a single male subject (1.86 m height, $80.00 \mathrm{~kg}$ weight, 28 years old, no previous history of back-pain) with no prior experience on exoskeleton usage. The subject was instructed to perform the following sequence of actions: lift a weight $(W, 11.4 \mathrm{~kg})$ from a surface (B1) at $0.5 \mathrm{~m}$ height from the ground (with self-chosen motion style); carry the weight for $3.5 \mathrm{~m}$ and lower it onto a second surface $((B 2))$ at $0.5 \mathrm{~m}$ height from the ground, returning to upright posture and resting in standing position. The object shape was designed in order not to offer evident handling points and to match industrial scenarios. Figure 1 summarizes the task description. The subject was required to perform this task as fast as possible and was given 10 seconds to complete the whole sequence. The task was repeated 10 times for each of the three following conditions:

- noExo: subject not wearing the exoskeleton;

- exoLift: subject wearing the exoskeleton constantly providing assistance;

- exoHar: subject wearing the exoskeleton switching between assistance and transparency based on HAR.

Section 2.2.4 reports more details about exoLift and exoHar conditions. No rest phase was planned between each of the 10 task repetitions, so that the faster the lifting-carrying-lowering sequence execution, the longer the standing phase duration. Following this $10 \mathrm{~s}$ interval, the subject was instructed to carry on with the next repetition. On the contrary, 5 minutes of rest were planned between the 3 conditions. The $11.4 \mathrm{~kg}$ weight was chosen so as to guarantee the task safety, according to revised NIOSH lifting equations ([22]) (NIOSH lifting index was 0.81 for males, and 0.99 for females), but trying to match realistic weight values lifted by industrial workers.

\subsubsection{Data acquisition.}

Efficacy. In order to assess efficacy, as proposed in Section 2.1, sEMG signals were recorded bilaterally at $1 \mathrm{KHz}$ sampling frequency from both Erector Spinae Longissimus and Ileocostalis muscles through surface electrodes (BTS FREEEMG, BTS Bioengineering, Italy). Electrodes placement was based on SENIAM guidelines ${ }^{1}$. Prior to the task execution start, maximum voluntary contraction (MVC) values for these muscles were acquired.

Dynamic fit. As presented in Section 2.1, dynamic fit is influenced by many factors. Here, the evaluation focuses only on those related to versatility (kinematic constraints, intuitiveness and ease of use, activity recognition performance). In order to assess kinematic metrics, motion data were collected, thanks to a 3D motion

\footnotetext{
${ }^{1}$ http://www.seniam.org/
} 
tracking system (MTw Awinda, Xsens, The Netherlands), at a $60 \mathrm{~Hz}$ sampling frequency. To track lower limbs and trunk movements, 8 inertial trackers were placed, respectively, on the sternum, the pelvis, right and left thighs, shanks and feet. Before the task start, a calibration of the Xsens Awinda system was performed. Moreover, during the task execution, the experimenter manually performed labelling of the activity carried out by the subject, classifying it into lifting, carrying or standing. To facilitate the manual labelling, the subject was required to spell out each of the activities he was performing. Both lifting and lowering tasks were classified as lifting for the purposes of the final data analysis. Manual activity labelling was used both for signal segmentation and for assessing the HAR performance of the exoskeleton, as indicated in Section 2.1. Acquisitions of sEMG signals, motion data, manual activity labelling and exoskeleton data were all synchronized. Even though presented in Section 2.1, in this case study, the subjective evaluation is omitted and only the device ability to manually or automatically switch between activities is assessed.

\subsubsection{Data processing.}

Efficacy. First, sEMG signals were band-pass filtered $(35-350 \mathrm{~Hz})$, smoothed and rectified in order to extract envelopes, and subsequently normalized with respect to MVC values. Second, the overall lumbar extensor activity was computed averaging the right and left side activity of Iliocostalis and Longissimus Lumborum muscles, as in [10]. Therefore, all the efficacy analysis is done with respect to this new variable. Third, the extensor activity was segmented based on the manual activity labelling performed during the experiment. Fourth, $90^{t h}$ and $50^{t h}$ percentiles of the EMG signal distribution were calculated for each lifting and carrying phases. Eventually, these metrics were further averaged over the 10 repetitions of lifting and carrying phase for each of the 3 conditions (noExo, exoLift, exoHar).

Dynamic fit. Kinematic data The joints under analysis are trunk, hip and knee. The angles of these joints were acquired through the Xsens system and segmented based on the manual activity labelling performed during the experiment. For each carrying segment, the initial and final $20 \%$ of the signals were discarded. This was done to consider only steady state gaits. Then, RoMs, TCTs and trunk mean inclination values were computed for each individual phase and, successively, averaged according to the condition. In this way, each metric and condition (noExo, exoLift and exoHar) was described by an average value. Furthermore, based on information about feet contacts with the ground provided by the XSens software, hip and knee joint angle profiles related to carrying phases were further segmented into single steps. This procedure was done to extract an average gait profile for each condition. To better analyse these trajectories, standard deviations and root mean square error (RMS) between the average gait profile in conditions with and without the exoskeleton were computed.

Exoskeleton data To evaluate activity recognition performances, the classification confusion matrix (CM) was extracted. The manual labelling was taken as the true class, whereas the activity recognized by the exoskeleton as the predicted one. Precision, recall and accuracy were then calculated. For each activity transition, the estimation time delay was computed as the difference between the instants in which automatic transition and the corresponding manual labelling occurred. The values obtained for transitions of the same type were, then, averaged.

2.2.4 The exoskeleton. The active back-support exoskeleton used in this study is XoTrunk (Figure 1), developed by the XoLab group at the Istituto Italiano di Tecnologia (IIT). XoTrunk, presented in [17], is a battery-powered occupational back-support exoskeleton. It can be worn like a backpack and delivers symmetrical extension torques at the hip joints applying forces through shoulder and thigh straps. The torques are generated by two brushless motors. Control of XoTrunk is structured in three levels. The low-level minimizes the error between the desired and the actual torque. More details about the implementation of this level, and about the actuators used to generate the torque can be found in [6]. The middle level modulates the desired torque relying on two different inputs: trunk inclination and myoelectric activity of the forearm ([20]). In this study, symmetric extensive torques were delivered according to the trunk flexion, saturating with a provision of $13 \mathrm{Nm}$ per side whenever it reached a $90^{\circ}$ inclination. Additional $13 \mathrm{Nm}$ were provided, per each side, as soon as the object was grabbed. Therefore, the maximum assistive torque of the exoskeleton was lower than $26 \mathrm{Nm}$ per side. Finally, the high level allows to recognize the activity performed by the user, based on a HAR algorithm presented in [13] and [14]. This level allows to adapt the assistance strategy to the activity performed by the user. In this study, XoTrunk was controlled according to two different strategies:

- exoLift: only middle and low levels of control are used. Thus, regardless of the activity performed, assistance is always computed combining trunk inclination and forearm activity. As a consequence, when performing carrying, even though the trunk inclination can be neglected, the exoskeleton still delivers assistance on the back and both hips, due to the forearm muscle activity, linked with weight handling.

- exoHar: all the three levels of control are used, and, so, the high level recognizes the activity. More in details, if a lifting or lowering activity is recognized, the exoskeleton provides assistance modulating the output torques via the two lower levels of control, as in exoLift; however, if a carrying activity is recognized, the exoskeleton enters a mode called transparency. This strategy commands for torques that generate null torque interaction between user and exoskeleton, so that no assistance is provided. As shown in [12], this strategy is expected not to hinder the natural gait.

\section{RESULTS AND DISCUSSION}

The average values obtained for the assessment metrics are summarized in Table 1 for lifting and in Table 2 for carrying. These results are presented in greater detail in the following sub-sections. Furthermore, for the sake of clarity, an interpretation is provided right after the results presentation. Overall discussions and considerations are presented in Section 4.

\subsection{Efficacy}

3.1.1 Lifting. Table 1 shows a reduction in muscular activity during lifting phases when wearing the exoskeleton. Indeed, in exoLift 

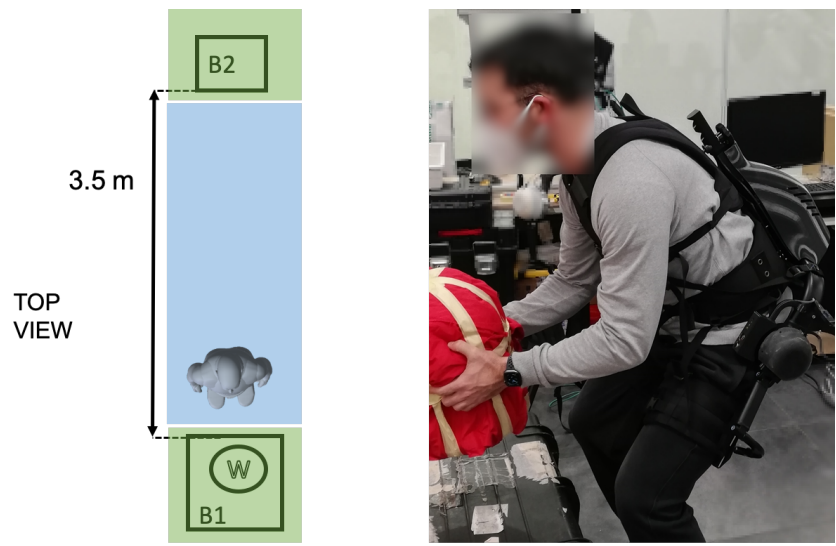

Figure 1: (Left) Schematic representation of the experimental task. $W$ represents the $11.4 \mathrm{~kg}$ weight, $B 1$ and $B 2$ are the two boxes onto which the weight was transported. Carrying task was performed within the light blue area $(3.5 \mathrm{~m})$ and lifting in the light green zones. Note that to move from $B 1$ to $B 2$ and viceversa, the subject needs to perform a $180^{\circ}$ rotation. (Right) A picture of the subject wearing XoTrunk and performing lifting. It is possible to note that the carried object $W$ did not offer evident handling points.

Table 1: Metrics average values during lifting

\begin{tabular}{|c|c|c|c|c|}
\hline \multicolumn{5}{|c|}{ Lifting } \\
\hline & & noExo & exoLift & exoHar \\
\hline \multirow{2}{*}{ Efficacy } & EMG $90^{\text {th }}(\%$ MVC $)$ & 33 & 27 & 28 \\
\hline & EMG $50^{t h}(\% M V C)$ & 26 & 18 & 21 \\
\hline \multirow{4}{*}{ Dynamic fit } & TCT (s) & 1.99 & 2.17 & 2.26 \\
\hline & Trunk inclination $\left({ }^{\circ}\right)$ & 60.64 & 56.37 & 51.69 \\
\hline & Hip RoM $\left(^{\circ}\right)$ & 70.90 & 59.83 & 54.72 \\
\hline & Knee RoM $\left(^{\circ}\right)$ & 46.61 & 44.81 & 36.19 \\
\hline
\end{tabular}

Table 2: Metrics average values during carrying

\begin{tabular}{|c|c|c|c|c|}
\hline \multicolumn{5}{|c|}{ Carrying } \\
\hline & & noExo & exoLift & exoHar \\
\hline \multirow{2}{*}{ Efficacy } & EMG $90^{\text {th }}(\%$ MVC $)$ & 27 & 18 & 20 \\
\hline & EMG $50^{t h}(\% M V C)$ & 19 & 12 & 15 \\
\hline \multirow{4}{*}{ Dynamic fit } & TCT (s) & 6.51 & 7.53 & 7.69 \\
\hline & Trunk inclination $\left(^{\circ}\right)$ & 5.00 & 10.83 & 10.73 \\
\hline & Hip RoM $\left(^{\circ}\right)$ & 34.84 & 26.78 & 27.02 \\
\hline & Knee RoM $\left(^{\circ}\right)$ & 46.63 & 41.86 & 43.24 \\
\hline
\end{tabular}

and exoHar conditions lower values were obtained with respect to the noExo one. In particular, the $90^{t h}$ percentile shows, with respect to the noExo condition a $19 \%$ reduction in exoLift, and a $17 \%$ reduction in exoHar. The $50^{\text {th }}$ percentile shows a $32 \%$ reduction in exoLift, and a $21 \%$ reduction in exoHar, again with respect to noExo.

Considering that during lifting, both exoLift and exoHar were exploiting the same inputs for the desired torque computation, the different reduction values might be related to variations in postures and forearm muscle activation. Computing an average of the two results, it is possible to state that the exoskeleton reduced the $50^{\text {th }}$ percentile by $27 \%$ and the $90^{t h}$ percentile by $18 \%$. These values are in line with those found in the literature [21].

3.1.2 Carrying. Table 2 reports that the $90^{\text {th }}$ percentile of EMG signal distribution during carrying is reduced with respect to the noExo condition both in exoLift (-32\%) and exoHar (-23\%). Reduction was lower in exoHar than in exoLift, but both conditions show a reduction with respect to noExo. The same happens for the $50^{t h}$ percentile as exoLift and exoHar display, respectively, a reduction by $37 \%$ and by $23 \%$. Again, reduction was lower in exoHar than in exoLift.

The exoLift condition results suggest that - considering only efficacy - assistance is beneficial even during carrying. Nonetheless, a reduction is also visible in exoHar (even though lower than in exoLift). These results differ from what found in a previous study, [12]. This represents a crucial aspect to be further investigated. Possible reasons that explain such difference could be due to changes in posture, a different shape adopted for the load to carry (and consequently different grasp techniques), benefits linked with the assistance provided during the previous lifting phase and the subsequent lowering phase.

\subsection{Dynamic fit}

\subsubsection{Time required to complete the task.}

Lifting. Average TCTs for lifting tasks were higher, with respect to noExo condition, when wearing the exoskeleton. Specifically, the values increased by $9 \%$ in exoLift, and by $13 \%$ in exoHar. The variation with respect to noExo condition was higher in exoHar than in exoLift, but both conditions show an increase with respect to noExo.

The slight increase of the average TCT for the lifting phase when wearing the exoskeleton - can partially be explained by the exoskeleton helping with gravity compensation, thus slowing down the lowering phase. Therefore, future works should investigate whether such an increase is acceptable in workplaces, or, on the contrary, negatively impacts productivity. In this study, the tested subject was always able to complete the sequence of tasks within the required $10 \mathrm{~s}$, so the exoskeleton did not interfere with task requirements.

Carrying. Average TCTs for carrying tasks were higher when wearing the exoskeleton. In particular, exoLift shows a $16 \%$ increase with respect to the noExo condition, whereas exoHar shows a $18 \%$ increase.

Being TCT, again, higher both in exoLift and exoHar conditions, without evident difference between the two, may suggest that the presence of the exoskeleton itself slows down movements, regardless of whether assistance is provided or not. However the fact that the subject is performing the activity slower may also indicate an improved confidence in handling the weight for a longer 
time. Indeed, it could indicate that the subject felt more comfortable when wearing the exoskeleton, and, thus, took the proper time to complete the task; on the contrary, in noExo condition the subject may have felt the need to speed up in order to lower the weight, as soon as possible. Further investigations may clarify these aspects.

\subsubsection{Trunk inclination.}

Lifting. With respect to the noExo test, during lifting activities, a reduction in trunk mean angle is visible in both exoLift and exoHar conditions. Specifically, exoLift shows a 7\% reduction, while exoHar shows a $15 \%$ reduction. The reduction is higher in the former condition. However, it should be taken into account that in this study both lifting and lowering were analyzed together. Future studies may distinguish between the two activities in order to look for more interesting results.

The reduction of trunk inclination in exoLift and exoHar conditions can be explained as a direct consequence of the exoskeleton assistance during lifting. Indeed, the provided torque always extends the back upwards. Moreover, as happened in this study, if it has no impact on the task execution, a reduced trunk inclination is better from an ergonomic viewpoint.

Carrying. Trunk average inclination during carrying were higher both in exoLift and exoHar conditions. In particular, exoLift shows a $117 \%$ increase with respect to noExo, while exoHar shows a $115 . \%$ increase. The obtained values are comparable and greater than $100 \%$.

The increase in trunk inclination when wearing the exoskeleton (regardless of whether it is performing HAR or not) may indicate that the exoskeleton itself induced the user to slightly bend when walking. However, further investigations may better clarify these results.

\subsubsection{Hip and knee joint angles and RoM.}

Lifting. Hip RoM was reduced both in exoLift and exoHar conditions with respect to noExo. Specifically, exoLift exhibits a $16 \%$ reduction with respect to noExo, while exoHar is reduced by $23 \%$. Also knee RoM was reduced both in exoLift and exoHar conditions with respect to noExo. Specifically, exoLift exhibits a $4 \%$ reduction with respect to noExo, while exoHar is reduced by $22 \%$. Both for hip and knee, the reduction is greater in exoHar than in exoLift. Hip and knee average angles profiles with standard deviation are visible in Figure 2. The analysis of Figure 2, shows that angles in noExo and exoLift assume close values both in hip and knee joints. Angles in exoHar assume lower values than in the other two conditions starting from approximately $15 \%$ of the activity. At the beginning of the movement, exoLift shows lower angle values with respect to noExo, while exoHar assumes intermediate values. From approximately $15 \%$ of the activity exoLift starts tracking noExo better than exoHar.

The fact that the RoM is reduced when wearing the exoskeleton might suggest that, thanks to the exoskeleton assistance, the subject needed to bend less. It would be interesting to study if this change of behaviour is due to the subject grabbing the object taking advantage of a different arm configuration. Furthermore, reduction was quite higher in exoHar, as clearly shown by the average joint angle profiles. This is an unexpected behaviour, since, in lifting, the exoskeleton is providing the same assistance both in exoLift and exoHar. In this work- for the sake of brevity - both lifting and lowering were analysed together. However, separating these two activities, could provide useful information to better clarify this aspect.
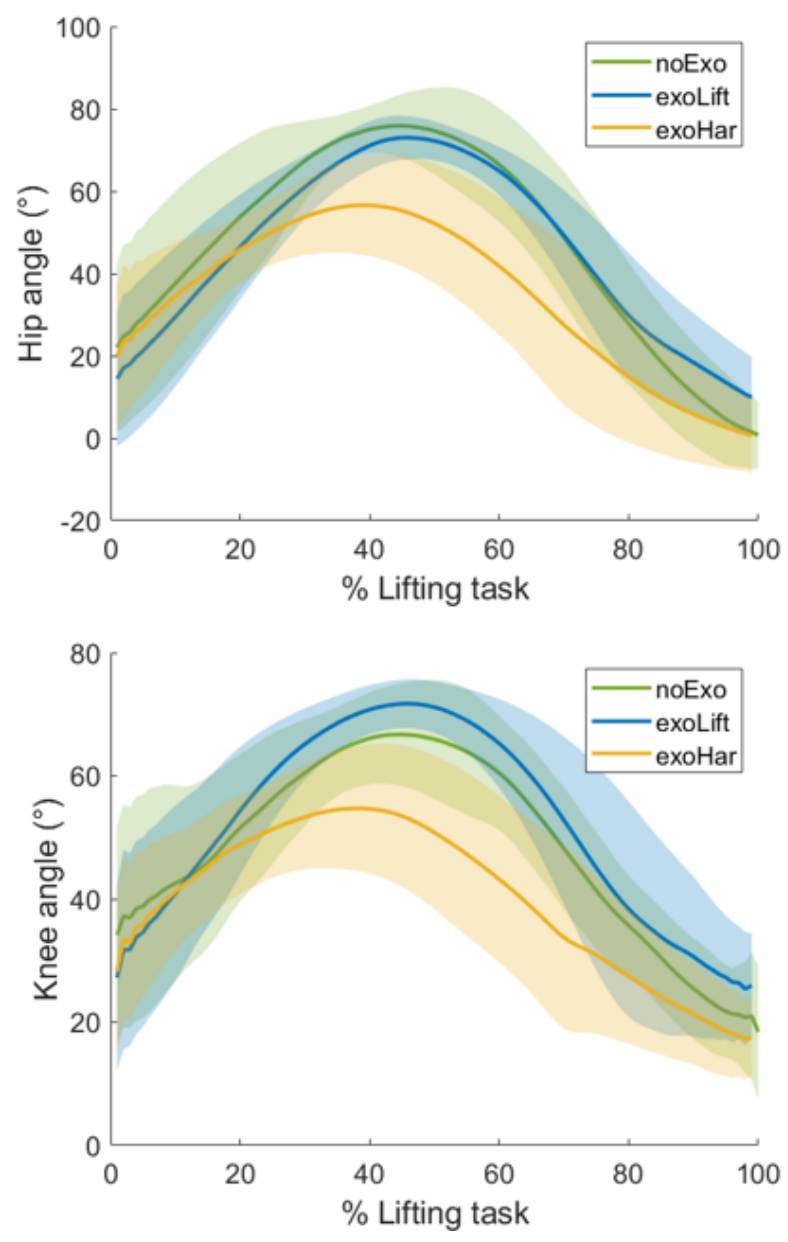

Figure 2: Hip (top) and knee (bottom) joint angles profiles during lifting activities. Solid lines represent average angle value, while shaded areas represent standard deviation (green for noExo, blue for exoLift, yellow for exoHar)

Carrying. With respect to noExo condition, wearing the exoskeleton reduced hip RoMs. In particular, exoLift generated a $23 \%$ reduction, while exoHar a $22 \%$ one. Reductions in these two conditions are comparable. Also the knee RoMs are reduced, with respect to noExo. Specifically, in exoLift by $10 \%$, while in exoHar by $7 \%$.

RoMs being comparable in both exoLift and exoHar is not what might be expected. In fact, as in exoHar the exoskeleton was not providing assistance during carrying, and thus there was no torque hampering the movements, it is safe to expect a higher RoM than in exoLift. This underlines that RoMs do not provide sufficient information and is why, here, it is suggested to further segment the carrying 
phase into single steps and consider the average step angle profiles. More in particular, analysing the average gait profiles (Figure 3) and noting the reduced flexion and the increased hyperextension of the joints, it is clear that exoLift was continuously extending both hip and knee joints. This might yield unnatural leg movements. Also, from Figure 3 it is evident that in exoLift, the profile is shifted towards negative values. On the contrary, exoHar presents a behaviour that is much closer to the noExo condition, as confirmed also by the RMS analysis. Indeed, RMS deviation between noExo and exoLift assumes a value of $4.78^{\circ}$ for the hip joint, and $4.99^{\circ}$ for the knee joint. RMS deviation between exoHar and noExo assumes a value of $1.36^{\circ}$ for the hip joint, and $3.48^{\circ}$ for the knee joint. Thus, less difference is visible between exoHar and noExo than between exoLift and noExo.

Considering the average gait trajectories, the closeness of the exoHar condition to the noExo one underlines how this control strategy appears to be more respectful of the wearer's natural movements. It also stresses how a strategy that ignores carrying actvities (as exoLift does) can have a negative impact on non-lifting tasks.

3.2.4 Intuitiveness and ease of use. XoTrunk is capable of automatic activity recognition and, thus, automatic selection of the most appropriate control strategy. This type of switch is expected to be better accepted by the user than a manual one, because it does not require user intervention in order to change control strategy. Indeed, a manual switch would imply the subject having to use at least one of his hands while holding a load.

3.2.5 Activity Recognition performance. The analysis of the performance is categorized according to the task. In particular, for the standing activity, an accuracy of $96.9 \%$, a precision of $93.8 \%$ and a recall of $90.4 \%$ were obtained; for lifting, accuracy of $90.7 \%$, precision of $91.3 \%$ and recall of $85.3 \%$ were obtained; and, eventually, for carrying, $88.7 \%$ accuracy, $87.2 \%$ precision and $98.7 \%$ recall were scored. The values obtained for the average estimation time delay show that automatic recognition is on average delayed with respect to manual labelling by $0.05 \mathrm{~s}$ for standing $\rightarrow$ lifting transition, by $0.01 \mathrm{~s}$ for carrying $\rightarrow$ lifting transition, and by $0.18 \mathrm{~s}$ for lifting $\rightarrow$ standing transition. The lifting $\rightarrow$ carrying transition, instead, is on average anticipated by $0.40 \mathrm{~s}$ with respect to manual labelling.

Classification performance indicate that the proposed HAR algorithm can be successfully used in complex task scenarios. Also, it is possible to note that standing recognition is more accurate than lifting and carrying. This implies that the algorithm might mislabel these two latter activities. A deeper analysis of the results should focus on when the errors occur. In particular, it is clear that if the classification error is within task transitions it has a much lower impact than at steady state. As an example, the consequences of wrongly setting assistance to zero are worse when the user is grabbing the load than when the bending movement is about to start. The anticipation of recognition in lifting $\rightarrow$ carrying transition might be explained by the fact that the subject started to spell the activity later than its actual beginning, and thus manual labelling was delayed with respect to automatic recognition. Furthermore, this transition occurred while the subject was performing a $180^{\circ}$ rotation in order to move from the starting position to the final one. The lifting $\rightarrow$ carrying transition shows the lowest delay value, indicating that it can be the easiest one to recognize.
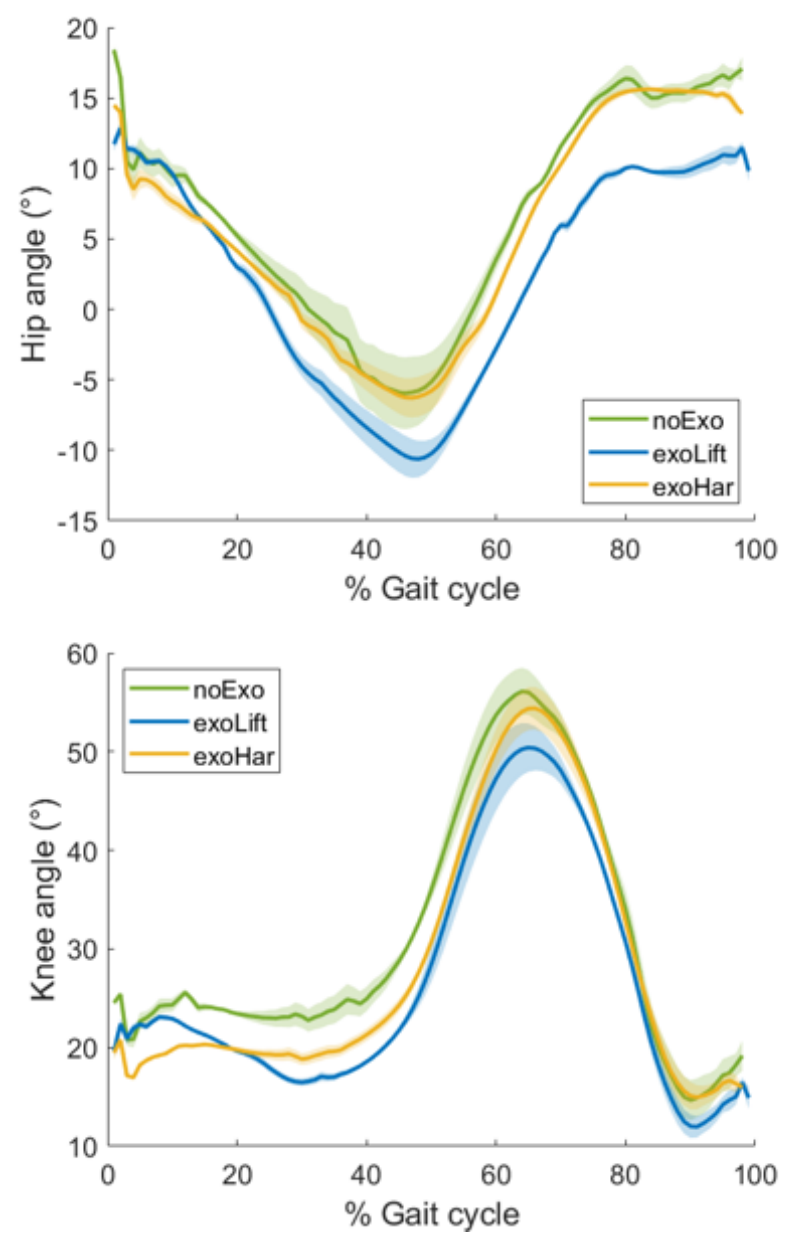

Figure 3: Right hip (top) and knee (bottom) joint angle profiles on a single step during carrying phase. Solid lines represent average angle value, while shaded areas represent standard deviation (green for noExo,blue for exoLift,yellow for exoHar)

\section{OVERALL CONSIDERATIONS}

First of all, it is worthy recalling that the results presented in this paper cannot be generalized, as they refer to a single subject. For this reason, no statistical analysis was performed, but it is still possible to make some considerations. In particular, focusing on efficacy, it is interesting to point out that the main effect of the device is an overall reduction of muscle activity and, to a smaller extent, a reduction in peak activity. The result suggests benefits in the prolonged usage of the device. Considering dynamic fit, the results underline that RoMs are not very informative and that average joint trajectories should be analysed, instead. Also, difficulties in the kinematic interpretation of the lifting data suggest that separating between lifting and lowering phases could guide the understanding of some of the open points. Moreover, the advantages of a versatile exoskeleton are not extremely evident if only efficacy is 
taken into account. Indeed, the analysis of efficacy metrics generally highlights muscle activity reduction, but does not capture the complexity of out of the lab environments. Considering also dynamic fit - on the contrary - allows to enlarge the dimensionality of the most appropriate exoskeleton selection and, in this situation, the advantage of active and quasi-passive devices over passive ones emerges. Indeed, the former devices have the possibility to better address versatility having the opportunity of modulating or removing assistance in non-lifting tasks, exploiting HAR. Of course, the performance of a versatile exoskeleton are strongly influenced by its capability of correctly classifying the activities being carried out. In this work, activity recognition performance appear adequate for usage in industry, although lifting recognition might require improvements. This could, in part, explain the different behaviour of the exoskeleton in lifting conditions.

\section{CONCLUSIONS}

The aim of this study was to describe a possible approach to investigate the effects of the usage of a versatile back-support exoskeleton in complex tasks. Versatility is obtained by means of Human Activity Recognition (HAR) algorithms that command for variable assistance strategy, according to the task. The analysis of exoskeleton versatility is expected to highlight how, in out-of the lab scenarios, the employment of active and quasi-passive exoskeletons can be an advantage with respect to passive devices. In this work, the analysis is done by evaluating not only the efficacy of an exoskeleton but also its dynamic fit. For such reason, first a list of relevant metrics were presented and, then, as an exemplification, the proposed analysis was applied to a case study involving a single subject performing a mixed combination of lifting, carrying and standing. The XoTrunk exoskeleton was used to compare the performance of a versatile control strategy and a non-versatile one. It was shown that the versatile control strategy, namely the one taking advantage of HAR, had an efficacy in lifting tasks (20\% back muscle reduction) comparable with the literature, and had lower impact on the workers natural movements during carrying. Nevertheless, to generalize the obtained results and investigate statistical significance, future studies should increase the sample of tested subjects. This extension could also help clarify open issues emerged in this study.

\section{ACKNOWLEDGMENTS}

This work was supported by the Italian Workers' Compensation Authority (INAIL) and by Istituto Italiano di Tecnologia (IIT) within the project Sistemi Cibernetici Collaborativi (SCC). Support was given, also, by the STREAM project funded by Shift2Rail Joint Undertaking, established under the European Union's Horizon 2020 framework programme for research and innovation, under grant agreement No 101015418.

\section{REFERENCES}

[1] SJ Baltrusch, JH Van Dieën, CAM Van Bennekom, and H Houdijk. 2018. The effect of a passive trunk exoskeleton on functional performance in healthy individuals. Applied ergonomics 72 (2018), 94-106.

[2] Don B Chaffin. 1969. A computerized biomechanical model-Development of and use in studying gross body actions. (1969).
[3] Baojun Chen, Lorenzo Grazi, Francesco Lanotte, Nicola Vitiello, and Simona Crea. 2018. A real-time lift detection strategy for a hip exoskeleton. Frontiers in neurorobotics 12 (2018), 17.

[4] Jan de Kok, P Vroonhof, J Snijders, et al. 2019. Work-Related musculoskeletal disorders: prevalence, costs and demographics in the EU: European risk Observatory report. Luxembourg: Publications Office of the European Union (2019).

[5] Michiel P De Looze, Tim Bosch, Frank Krause, Konrad S Stadler, and Leonard W O'Sullivan. 2016. Exoskeletons for industrial application and their potential effects on physical work load. Ergonomics 59, 5 (2016), 671-681.

[6] Christian Di Natali, Stefano Toxiri, Stefanos Ioakeimidis, Darwin G Caldwell, and Jesús Ortiz. 2020. Systematic framework for performance evaluation of exoskeleton actuators. Wearable Technologies 1 (2020).

[7] Lorenzo Grazi, Baojun Chen, Francesco Lanotte, Nicola Vitiello, and Simona Crea. 2019. Towards methodology and metrics for assessing lumbar exoskeletons in industrial applications. In 2019 II Workshop on Metrology for Industry 4.0 and IoT (MetroInd4. 0\&IoT). IEEE, 400-404.

[8] Marko Jamšek, Tadej Petrič, and Jan Babič. 2020. Gaussian Mixture Models for Control of Quasi-Passive Spinal Exoskeletons. Sensors 20, 9 (2020), 2705.

[9] Bengt Jonsson. 1982. Measurement and evaluation of local muscular strain in the shoulder during constrained work. fournal of human ergology 11, 1 (1982), 73-88.

[10] Axel S Koopman, Idsart Kingma, Gert S Faber, Michiel P de Looze, and Jaap H van Dieën. 2019. Effects of a passive exoskeleton on the mechanical loading of the low back in static holding tasks. fournal of biomechanics 83 (2019), 97-103.

[11] Pamela V Moore and Arun Garg. 1995. Revised NIOSH equation for manual lifting: a method for job evaluation. AAOHN fournal 43, 4 (1995), 211-218.

[12] Tommaso Poliero, Maria Lazzaroni, Stefano Toxiri, Christian Di Natali, Darwin Gordon Caldwell, and Jesus Ortiz. 2020. Applicability of an active backsupport exoskeleton to carrying activities. Frontiers in Robotics and AI 7 (2020), 157.

[13] Tommaso Poliero, Lorenza Mancini, Darwin G Caldwell, and Jesús Ortiz. 2019. Enhancing Back-Support Exoskeleton Versatility based on Human Activity Recognition. In 2019 Wearable Robotics Association Conference (WearRAcon). IEEE, 86-91.

[14] Tommaso Poliero, Stefano Toxiri, Sara Anastasi, Luigi Monica, and Darwin G Caldwelll Jesús Ortiz. 2019. Assessment of an On-board Classifier for Activity Recognition on an Active Back-Support Exoskeleton. In 2019 IEEE 16th International Conference on Rehabilitation Robotics (ICORR). IEEE, 559-564.

[15] Linda Shore, Valerie Power, Bernard Hartigan, Samuel Schülein, Eveline Graf, Adam de Eyto, and Leonard O'Sullivan. 2020. Exoscore: A design tool to evaluate factors associated with technology acceptance of soft lower limb exosuits by older adults. Human Factors 62, 3 (2020), 391-410.

[16] Matteo Sposito, Darwin G Caldwell, Elena De Momi, and Jesús Ortiz. 2020 (not published yet). Subjective assessment of Occupational Exoskeletons: feasibility study for a Custom Survey for Braces. In International Symposium on Wearable Robotics. Springer.

[17] Matteo Sposito, Christian Di Natali, Stefano Toxiri, Darwin G Caldwell, Elena De Momi, and Jesús Ortiz. 2020. Exoskeleton kinematic design robustness: An assessment method to account for human variability. Wearable Technologies 1 (2020).

[18] Leia Stirling, Damian Kelty-Stephen, Richard Fineman, Monica LH Jones, ByoungKeon Daniel Park, Matthew P Reed, Joseph Parham, and Hyeg Joo Choi. 2020. Static, dynamic, and cognitive fit of exosystems for the human operator. Human Factors 62, 3 (2020), 424-440.

[19] Jean Theurel and Kevin Desbrosses. 2019. Occupational exoskeletons: Overview of their benefits and limitations in preventing work-related musculoskeletal disorders. IISE Transactions on Occupational Ergonomics and Human Factors 7, 3-4 (2019), 264-280.

[20] Stefano Toxiri, Axel S Koopman, Maria Lazzaroni, Jesús Ortiz, Valerie Power, Michiel P de Looze, Leonard O'Sullivan, and Darwin G Caldwell. 2018. Rationale, implementation and evaluation of assistive strategies for an active back-support exoskeleton. Frontiers in Robotics and AI 5 (2018), 53.

[21] Stefano Toxiri, Matthias B Näf, Maria Lazzaroni, Jorge Fernández, Matteo Sposito, Tommaso Poliero, Luigi Monica, Sara Anastasi, Darwin G Caldwell, and Jesús Ortiz. 2019. Back-support exoskeletons for occupational use: an overview of technological advances and trends. IISE Transactions on Occupational Ergonomics and Human Factors 7, 3-4 (2019), 237-249.

[22] Thomas R Waters, Vern Putz-Anderson, Arun Garg, and Lawrence J Fine. 1993. Revised NIOSH equation for the design and evaluation of manual lifting tasks. Ergonomics 36, 7 (1993), 749-776.

[23] Andrea Maria Zanchettin, Elizabeth Croft, Hao Ding, and Miao Li. 2018. Collaborative robots in the workplace [from the guest editors]. IEEE Robotics \& Automation Magazine 25, 2 (2018), 16-17. 